\title{
Resistance in Escherichia coli after single dose ampicillin to treat gonorrhoea
}

\author{
F M MULCAHY,* C J LACEY,* K BARR, $†$ AND R W LACEY $\S$ \\ From the *Department of Genitourinary Medicine, General Infirmary, Leeds, and the †Department of \\ Microbiology, University of Leeds, Leeds
}

SUMMARY Patients with uncomplicated gonorrhoea were treated with a single dose of either ampicillin $3 \mathrm{~g}$ orally or procaine penicillin $2.4 \mathrm{MIU}$ by injection, both with probenecid $1 \mathrm{~g}$ orally. The proportion of faecal Escherichia coli resistant to ampicillin before and a week after treatment was assessed. Of 55 patients treated with ampicillin who initially possessed sensitive flora, $25(45 \cdot 5 \%)$ became colonised subsequently by resistant $E$ coli. Resistance to ampicillin, together with resistance to some other antimicrobials, was tranferable in vitro. Penicillin, however, selected resistant $E$ coli in only four $(14.3 \%)$ out of 28 patients with initially sensitive flora. There was no difference in therapeutic response between the two agents. Intramuscular penicillin appeared to offer appreciably greater microbiological benefits than oral ampicillan in treating gonorrhoea.

\section{Introduction}

Acquired resistance to antimicrobials arises predominantly by the accidental exposure of commensal bacteria to antimicrobials, ${ }^{1}$ rather than by alterations of the pathogen during treatment. Most resistance to antimicrobials has been generated by the use of penicillins that select for organisms that produce $B$ lactamases. Among Gram negative bacteria $R$ factors are largely responsible for coding $B$ lactamases, which can transfer from one culture or species to another. Selection of resistant bacteria in the gut flora after treatment with antimicrobials may have considerable long term consequences. Most urinary tract infections are caused by commensal bacteria of the gastrointestinal tract. The origins of $B$ lactamase in gonococci may also be in the commensal flora of the respiratory or intestinal tract.

Though $B$ lactam antimicrobials given for one week are known to select resistance in the gut flora, ${ }^{2}$ there is a belief that single dose treatment is not particularly liable to select resistance. ${ }^{34}$ Uncomplicated gonorrhoea is often treated in the United Kingdom with either a single dose of oral ampicillin and probenecid or intramuscular procaine penicillin and oral probenecid. The decision is based on considerations of

Address for reprints: Dr FM Mulcahy, Department of Genitourinary Medicine, Blundell Street Clinic, The General Infirmary at Leeds, Great George Street, Leeds LS1 3EX

Accepted for publication 12 September 1985 convenience and compliance, with little emphasis placed on risks of selection of resistance. We therefore examined the effect of these two single dose regimens on the flora of the gut.

\section{Patients and methods}

\section{PATIENTS}

We studied consecutively 55 men and 63 women with uncomplicated gonorrhoea at the departments of genitourinary medicine at the General Infirmary, Leeds, and St Luke's Hospital, Bradford. Patients describing penicillin hypersensitivity, contact with Chlamydia trachomatis or any other sexually transmitted infection requiring further antibiotic treatment, or who had used antimicrobials within the previous month were excluded.

Patients (75) who attended the General Infirmary at Leeds were treated with ampicillin $3 \mathrm{~g}$ and probenecid $1 \mathrm{~g}$ orally, while those attending St Luke's Hospital (43 patients) were treated with intramuscular procaine penicillin $2.4 \mathrm{MIU}$ and oral probenecid $1 \mathrm{~g}$. These treatment regimens complied with the general policies of each unit, and though treatment might ideally have been randomised, this was not feasible. Sex distribution, age range, and demographic characteristics were similar in both groups of patients. Rectal swabs were taken and transported in Stuart's medium from all patients before treatment. The mean transit times between the clinics in Leeds and Bradford and the laboratory were 24 and 48 hours (ranges two to 48 
hours and 24 to 72 hours) respectively. Material for smears and cultures to diagnose gonorrhoea was taken from the urethra and cervix of women and the urethra of heterosexual men. Material for cultures was also taken from the urethra, rectum, and pharynx and for smears from the urethra and rectum of homosexual men. The diagnosis of gonorrhoea was based on recognition of Gram negative diplococci in smears, and was confirmed by culture of Neisseria gonorrhoeae in all patients. Patients were reviewed one week after treatment when material for smears and cultures was again taken from all relevant sites including the rectum.

\section{METHODS}

$N$ gonorrhoeae was cultured by conventional methods, including the test to detect $B$ lactamase.

To evaluate resistance to ampicillin of Escherichia coli, each rectal swab was shaken in sterile water and viable counts derived on antibiotic free medium and medium containing $100 \mathrm{mg}$ ampicillin/l. Rectal swabs were taken because of the difficulty of obtaining faecal samples from these patients. Viable counts were obtained by diluting the emulsified rectal swabs with distilled water to $10^{-1} / \mathrm{ml}, 10^{-2} / \mathrm{ml}, 10^{-3} / \mathrm{ml}$, and $10^{-4} / \mathrm{ml}$ and by applying $0.1 \mathrm{ml}$ aliquots to the surface of antibiotic free medium or the same medium containing $100 \mathrm{mg}$ ampicillin/l. The identification of a proportion of the presumptive $E$ coli was confirmed by API 20E (API Ltd, Basingstoke, Hampshire). Resistance to other antibiotics was assessed initially by disc testing and confirmed by estimation of minimum inhibitory concentrations. ${ }^{2}$

Each $E$ coli culture resistant to ampicillin was incubated with $E$ coli UB5201 to test for transferability of the resistance. ${ }^{6}$ Appropriate controls were always included. ${ }^{7}$ Successful transfer was defined as the presence of resistance to ampicillin in the recipient at an incidence of $>10^{-8}$ resistant recipients out of total donors.

Plasmid deoxyribonucleic acid was identified in a proportion of the transconjugants using the method of Birnboim and Doly. ${ }^{8}$

\section{Results}

All patients who received either ampicillin or procaine penicillin were cured of gonorrhoea clinically after a week, and cultures were then negative for this organism. None of the isolates produced $B$ lactamase. Table I shows that out of 75 patients treated with ampicillin and probenecid, $20(26.7 \%)$ initially possessed a high incidence (more than $10^{-2}$ ) of ampicillin resistant $E$ coli in their gut flora. Except in one patient, the resistance persisted after a week. Of the 55 patients who possessed no detectable ampicillin resistance (less than $\left.10^{-4}\right)$ before treatment, $25(45 \cdot 5 \%)$ harboured ampicillin resistant $E$ coli (more than $10^{-2}$ ) a week after the treatment with a single dose of ampicillin $3 \mathrm{~g}$.

Of 43 patients treated with procaine penicillin, 15 (34.9\%) initially had ampicillin resistant $E$ coli in the gut flora. There was no significant difference between the two groups of patients with initially ampicillin resistant $E$ coli $\left(\chi^{2}=2 \cdot 03 ; \mathrm{p}>0 \cdot 1\right)$. In contrast to the ampicillin treated group, however, only four patients acquired resistance to ampicillin in $E$ coli after treatment with procaine penicillin and two lost resistance. The net effect of procaine penicillin on resistance to ampicillin was thus a gain of resistance in

TABLE I Selected resistance to ampicillin in Escherichia coli in 118 patients with gonorrhoea treated by two different single dose regimens.

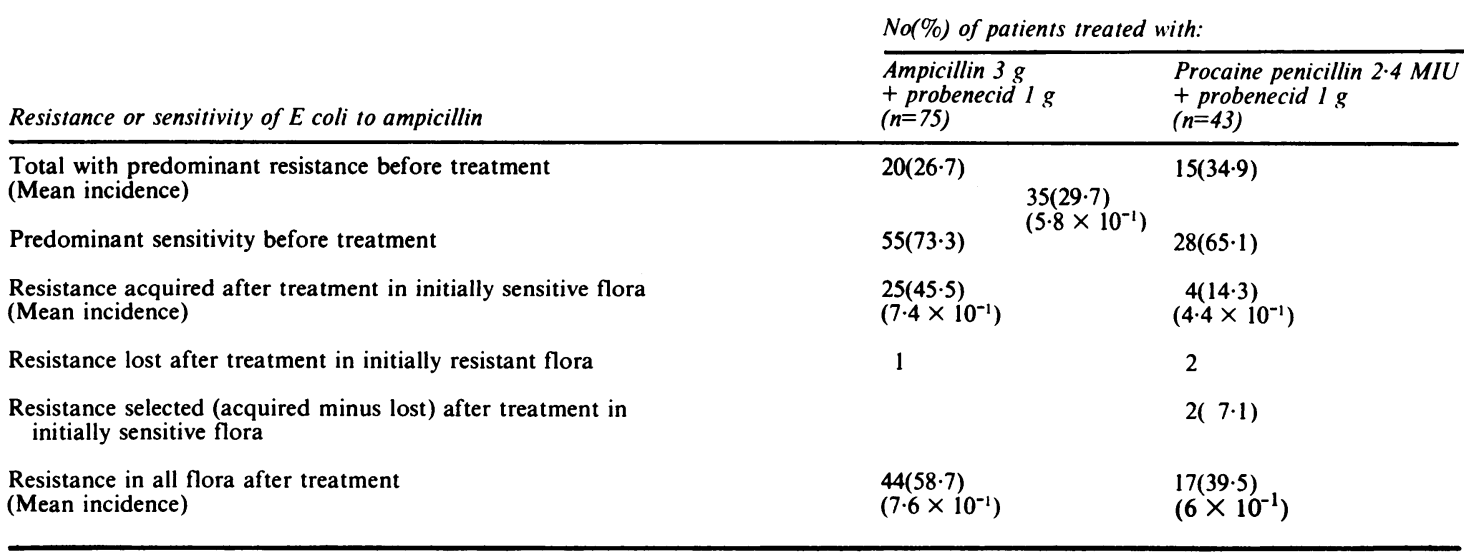


TABLE II Cross resistance to six other antimicrobials of 21 strains of Escherichia coli with selected resistance to ampicillin (resistance defined by minimum inhibitory concentrations of antimicrobials)

\begin{tabular}{lll}
\hline & \multicolumn{1}{l}{ No(\%) treated with: } \\
\cline { 2 - 3 } $\begin{array}{l}\text { Antimicrobial } \\
\text { agent }\end{array}$ & $\begin{array}{l}\text { Ampicillin } \\
(n=21)\end{array}$ & $\begin{array}{l}\text { Procaine penicillin } \\
(n=4)\end{array}$ \\
\hline $\begin{array}{l}\text { Sulphonamide } \\
(\mathrm{MIC}>200 \mathrm{mg} / \mathrm{l})\end{array}$ & $16(76)$ & $1(25)$ \\
$\begin{array}{l}\text { Tetracycline } \\
\text { (MIC }>25 \mathrm{mg} / \mathrm{l})\end{array}$ & $11(52)$ & $1(25)$ \\
$\begin{array}{l}\text { Cephradine } \\
(\mathrm{MIC}>100 \mathrm{mg} / \mathrm{l})\end{array}$ & $3(14)$ & 0 \\
$\begin{array}{l}\text { Trimethoprim } \\
(\mathrm{MIC}>5 \mathrm{mg} / \mathrm{l})\end{array}$ & 0 & 0 \\
$\begin{array}{l}\text { Gentamicin } \\
(\mathrm{MIC}>10 \mathrm{mg} / \mathrm{l})\end{array}$ & 0 & 0 \\
$\begin{array}{l}\text { Streptomycin } \\
(\mathrm{MIC}>25 \mathrm{mg} / \mathrm{l})\end{array}$ & $10(48)$ & 0 \\
\hline
\end{tabular}

two out of 28 patients. Differences between the effect of ampicillin and procaine penicillin in $E$ coli selecting ampicillin resistance (expressed as patients with resistance of more than $10^{-2}$ present) was highly significant $\left(\chi^{2}=9.48 ; p<0.01\right)$.

Twenty one of the ampicillin resistant strains of $\boldsymbol{E}$ coli appearing after treatment were available for study. Table II shows that they had a high incidence of resistance to sulphonamide (76\%), tetracycline (52\%), and streptomycin (48\%).

In 12 strains $(57 \%)$ resistance to ampicillin was transferable (mean incidence $8.8 \times 10^{-3}$; range $5 \times 10^{-2}$ to $\left.1.4 \times 10^{-7}\right)$. Resistance to sulphonamide was transferred with that to ampicillin in three strains, to tetracycline in one strain, and to sulphonamide and tetracycline together in one strain. Similarly, resistance to ampicillin in $E$ coli obtained after treatment with procaine penicillin was transferable in all four instances (mean incidence $2.3 \times 10^{-2}$; range 5.7 $\times 10^{-2}$ to $\left.2 \times 10^{-4}\right)$.

\section{Discussion}

The main difference between the effect of ampicillin and procaine penicillin was the propensity of ampicillin to select $E$ coli resistant to itself and also to other antibiotics. No differences in efficacy against $\boldsymbol{N}$ gonorrhoeae were seen, as there were no therapeutic failures with either regimen. Though the administration of $B$ lactam antibiotics for one week is known to select resistance in $E$ coli, the propensity of a single dose of ampicillin to select resistance is disturbing. In mice, the administration of ampicillin can replace the sensitive gut flora with resistant strains in 24 to 48 hours. ${ }^{9}$ Our results amplify those of Percival and Hart, who in a small study showed that ampicillin rather than penicillin selected resistant coliforms in the intestine. ${ }^{10}$

The reason that ampicillins exert a potent selection pressure in the intestine is probably multifactorial: their poor absorption from the gastrointestinal tract and their wide range of antimicrobial activity may be important. The opportunity of the extrachromosomal genetic determinants to "spread" to gut organisms with good colonising ability may also contribute. Certainly transfer of resistance to ampicillin occurs between enterobacteriaceae in volunteers who receive ampicillin. ${ }^{11}$ That ampicillin resistance was often linked with tetracycline resistance suggests that prior tetracycline treatment may have also played a part in the formation of these plasmids. Not surprisingly, preliminary data from a study investigating the effect of a one week course of tetracycline on the gut flora after treatment of Chlamydia trachomatis confirms the ability of tetracycline to select resistance to itself and to other antibiotics, notably ampicillin (Mulcahy FM, Lacey RW, data not published). The long term persistence of ampicillin resistant bacteria in the intestine is unpredictable. Though we were unable to establish the mean duration of persistance of selected resistant coliforms in this patient population because of their non-compliance with long term follow up, other studies have shown that these organisms, once selected, can remain for some months. ${ }^{12}$

In our laboratory, $38 \%$ of all cultures of $E$ coli isolated from fresh clinical material are resistant to ampicillin. Apart from being used to treat gonorrhoea, ampicillin (or amoxycillin) is also being used for a variety of infections when a narrow range antibiotic, such as penicillin, would seem preferable - certainly from the point of view of selection of resistance.

Some clinics prefer to use ampicillin (or amoxycillin) rather than injectable penicillins to treat gonorrhoea because oral treatment is easier to administer and has a lower incidence of anaphylaxis. ${ }^{13}$ Indeed, the selection of resistance to ampicillin in commensal bacteria has little immediate relevance to the patient in question. We stress, however, that such resistant bacteria contain a reservoir of resistance determinants that may become incorporated into several pathogenic bacteria, including $N$ gonorrhoeae. For these reasons, we recommend that when penicillin is indicated for treating gonorrhoea (and some other infections) a narrow range penicillin, such as procaine penicillin, is used rather than ampicillin.

We thank Drs M A Waugh and K C Mohanty for permission to report on their patients. 


\section{References}

1. Datta N. Antibiotic resistance in bacteria. Br Med Bull 1984;40:1-3.

2. Lacey RW, Lord VL, Howson GL, et al. Double blind study to compute the selection of antibiotic resistance by amoxycillin or cephradine in commensal flora. Lancet 1983;ii:529-32.

3. Anderson JD, Aird MY, Johnson AM, et al. The use of a single 1 gram dose of amoxycillin for the treatment of acute urinary tract infections. J Antimicrob Chemother 1979;5:481-3.

4. Lacey RW, Simpsom MHC, Lord VL, et al. Comparison of single-dose trimethoprim with a five-day course for the treatment of urinary tract infections in the elderly. Age Ageing 1981;10:179-85

5. Percival A, Rowlands J, Corkill JE, et al. Penicillinase producing gonococci in Liverpool. Lancet 1976;ii:1379-82.

6. Sanchez J, Bennet P M, Richmond M H. Expression of elt-B, the gene encoding the $B$ unit of the heat labile enteroxin of Escherichia coli when cloned in p ACYC 184. FEMS Microbiology Letters 1982;14:1-5.
7. Bennet PM, Richmond MH. Translocation of a discrete piece of deoxyribonucleic acid carrying an ampicillin gene between replicons in Eschirichia coli. J Bacteriol 1976;126:1-6.

8. Birnboim HC, Doly J. A rapid alkaline extraction procedure for screening recombinant plasmid DNA. Nucleic Acids Res 1979;7:1513-23.

9. Schaedler RW, Warren GH. Effect of cyclacillin and ampicillin on the gut flora of mice. Chemotherapy 1980;26:289-96.

10. Percival A, Hart CA. Rationale for antimicrobial therapy of infections caused by multiply resistant Neisseria gonorrhoeae. In: Grooks GF, ed. Immunology of Neisseria gonorrhoeae. Washington DC: American Society for Microbiology, 1978,805 .

11. Anderson JD, Gillespie WA, Richmond HC. Chemotherapy and antibiotic resistance transfer between enterobacteria in the human gastrointestinal tract. J Med Microbiol 1973;6:461-73.

12. Hartley $\mathbf{C L}$, Richmond $\mathbf{M H}$. Antibiotic resistance and survival of $E$ coli in the alimentary tract. $B r$ Med J 1975;iv:71-4.

13. Erffmeyer JE. Adverse reactions to penicillin. Ann Allergy $1981: 47: 288-93$ 